Dr. A. Seeligmiller, Ein Fall von einer auf den Nervus cutaneus etc. 575

\title{
XXI.
}

\section{Ein Fall von einer auf den Nervus cutaneus brachii internus minor beschränkten Neuralgie.}

\author{
Von \\ Dr. A. Seeligmüller, \\ in Halle a./S.
}

Frau Wilhelmine B., 43 Jahr alt, aus R., hat 8 lebendige Kinder geboren (eins ist gestorben, das jüngste ist $1 \frac{1 / 2}{2} \mathrm{Jahr}$ alt), alle selbst genährt, das jüngste 6 Monate lang. Im 21. Lebensjahre überstand sie einen Typhus, seitdem sind die Kopfschmerzen, an welchen sie vorher gelitten, viel seltener geworden; sonst war sie stets gesund.

Ihr jetziges Leiden bat sie vor 8 Jahren bekommen, als sie im dritten Monat schwanger war. Sie wohnte damals in einer sehr feuchten Wohnung.

Zunächst stellte sich ein schmerzhaftes Brennen in der linken Achselhöhle ein, welches dieselbe Enpfindung darstellte, als ob ein brennender Spahn dahin gehalten würde. Dieses Brennen hielt gewöhnlich eine halbe bis eine Stunde lang an und wiederholte sich anfangs nur alle 2 bis 4 Wochen, später kam es häufiger. Nachdem diese Anfälle von Brennen ein Jahr lang bestanden, gesellte sich zu demselben ein plötzlich den linken Oberarm und die Schulter durchzuckender Schmerz. Dieser beginnt auch jetzt noch dicht oberhalb des Olecranon, fährt von da aus nach dem hinteren Theil der Achselhöble und von hier aus quer uber den unteren Winkel des linken Schulterblatts: alles dies so schnell, dass es voruber ist, ehe Patientin darüber sprechen kann. Ausserdem schiesst der Schmerz zum Schluss nicht selten von der Schulter in die linke Brust hinein. Dabei hat sie dann dieselbe Empfindung "wie beim Stillen in dem Augenblick, wo die Nahrung in die Brust hineinschiesst." $\mathrm{Zu}$ bemerken ist noch, dass, obgleich sie das Kind schon seit 10 Monaten entwöhnt hat, beide Bruste doch noch Milch secerniren.

Dieser letztgeschilderte schiessende Schmerz wiederholt sich bei Tag und Nacht sehr hăufig und hat sie während der letzten 7 Jahre nie auf längere Zeit verlassen. Nachts wird sie dadurch nicht selten aus dem Schlafe geweckt.

Besonders heftig trat er auf, so oft sie schwanger war, ebenso wie auch jetzt noch, zur Zeit der Regel. Ausserdem empfindet sie fortwahrend in der- 
selben Bahn einen fast continuirlichen, aber viel weniger heftigen und darum erträglicheren Schmerz.

Das früher allein vorhandene Brennen in der Achselhöhle hat sich seit 4 Jahren mehr verloren, kommt aber zeitweise immer wieder einmal noch mit hinzu. Sind die Schmerzen sehr heftig, so soll sich der ganze Arm zu* weilen ganz kalt anfühlen.

Seit 4 Wochen ist der Schmerz besonders heftig; während dieser Zeit hat sie nur wenig essen können; der Stuhl ist regelmässig. Iie Regel kommt zuweilen 8 Tage zu spät, seltener 14 Tage.

Mannigfache Curversuche, auch ein solcher mit dem constanten Strom waren durchaus erfolglos gewesen.

\section{Status praesens vom 28. Mai 1875.}

Patientin ist eine grosse, mehr hagere, brünette Frau, welche für ihre Jahre und die acht Geburten noch recht gut aussieht.

Der linke Oberarm und die linke Schulter sind vielleicht ein klein wenig magerer als die betreffenden Theile rechts. Es lassen sich in der oben beschriebenen Schmerzensbahn nirgends Schmerzdruckpunkte finden. Dagegen ist die Gegend nach links von den Dornfortsätzen des letzten Hals- und des ersten Brustwirbels bei tiefern Druck ziemlich empfindlich.

Da der Schmerz in dem vorliegenden Falle ungefähr in der Bahn des Nervus ulnaris verlief, so bätte man an eine Neuralgie dieses so häufig neuralgisch afficirten Nerven denken können. Weil die Kranke aber die Schmerzempfindung nur bis zum Olecranon hatte und vor allem jede abnorme Sensation in den beiden letzten Fingern sowohl während als ausser den Anfällen mit Bestimmtheit in Abrede stellte, so musste eine Neuralgia ulnaris mit Sicherheit ausgeschlossen werden. Vielmehr konnte es sich nur um eine Neuralgie des Nervus cutaneus internus minor s. N. cutan. medialis (Henle) handeln.

Mit dieser Annahme stimmten zunächst die Angaben der Kranken über die Bahn, in welchor die Schmerzen liefen, auf das Genaueste überein. Der genannte kurze Hantnerv, über dessen genauere Anatomie das bekannte Henle'sche Lehrbuch im Text auf Seite 481, sowie durch die Figuren 270, 271, 272, 281 und 282 genauere Auskunft giebt, ist seinem Ursprung nach der am meisten nach unten gelegene Zweig des Plexus brachialis. Diesem Umstande entspricht in unserem Falle der Schmerzdruckpunkt zur linkeu Seite der Dornfortsätze des letzten Hals- und ersten Brustwirbels. Er giebt aus der Achselgrube einen Zweig nach hinten ab, welcher sich um die Sehne des M. latissimus dorsi herum aufwärts zur Scapulargegend schlägt. Daher der gewöhnlich nach dem Schulterblatt hinstrablende Schmerz. Auch die Angabe der Kranken, dass der Schmerz bisweilen bis in die linke Mamma hinein sich erstreckt, hat eine sichere anatomische Grundlage. Unser Hautnerv steht nämlich in sehr enger Beziehung zu dem lateralen Hautaste des zweiten Intercostalnerven, durch welchen er vollständig ersetzt werden oder aber mit welchem er sich zu einem Stämmchen vereinigen kann. Von der Richtigkeit dieser Angaben Henle's habe ich mich selbst zum Ueberfluss an Spirituspräparaten der hiesigen Anatomie hinreichend überzengt.

Schliesslich sei noch erwähnt, dass die Kranke in dem ganzen Gebiete 
Ein Fall von einer auf den Nerv, cutanens brachii internus minor etc. 577

des Nv. cutaneus medialis von dem Olecranon bis zur Scapula den faradischen Pinsel bei einer gewissen Stromstärke weniger heftig fühlte, als an den benachbarten Hautstrecken desselben oder den entsprechenden des anderen Armes. Eine allerdings erst später, als schon eine bedeutende Besserung der Neuralgie eingetreten war, vorgenommene Prufung mit dem Tasterzirkel ergab keine bemerkbaren Unterschiede.

Ueber den weiteren Verlauf noch Folgendes: Nachdem sich mehrmalige Behandlungen mit dem faradischen Pinsel als vollständig erfolglos, Morphium subcutan aber nur von schnell vorübergehendem Erfolge gezeigt hatten, führte die Behandlung mit dem Batteriestrome sehr schnell und sicher zu einem günstigen Resultate. Der negative Pol wurde auf deu oben bezeichneten Schmerzdruckpunkt seitlich von dem letzten Hals- und ersten Brustwirbel, der positive aber oberhalb des Olecranon oder auch auf die Mitte des Oberarms aufgesetzt und so mehr oder weniger die ganze Länge des Nerven in den Strom eingeschaltet ( 40 grosse Siemens-Halske'sche Elemente, 6 bis 10 Minuten lang; sorgfältiges Ein- und Ausschleichen des Stromes.) Nach der ersten derirtigen Sitzung war Patientin seit acht Jahren zum ersten Male 24 Stunden hintereinander frei von allen Schmerzen. Leider musste, nachdem die Behandlung kaum acht Tage lang mit fast demselben constanteu Erfolge fortgesetzt war, dieselbe fast 3 Wochen lang wegen eines starken Gesichtserysipels unterbrochen werden. In den ersten 6 Tagen diescr intercurrenten Krankheit war Pationtin zum Staunen ihrer Angehörigen, zu denen sie gereist war, vollständig frei von allen Schmerzen im Arme geblieben und auch dann stellte sich nur von Zeit zu Zeit cin leichtes Reissen in demselben ein. Erst 2 Tage vor ihrer Rückkehr zur electrischen Cur traten die Schmerzen wieder einmal heftiger auf. Patientin schien jetzt nicht mehr so starke Ströne wie fraher $\mathbf{z u}$ vertrageu, fand aber dann durch schwächere Ströme wieder dieselbe Erleichterung wie früher. Nach weiterer achttägiger Behandlung kehrte Patientin sehr erfreut über das erzielte Resultat in ihre Heimath zurück. Ob das letztere von Dauer sein wird, ist abzuwarten.

In dem eben beschriebenen Fall einer auf den Nervus cutaneus medialis allein beschränkten Neuralgie dürte kaum eiu zweiter analoger in der Literatur gefunden werden. Wenigstens ist es mir bis jetzt nicht gelungen, obgleich ich ausser den Lebrbüchern der Nerrenkrankheiten von Romberg, Hasse, Eulenburg, Jones, Rosenthal etc. auch die Monographie uber Neuralgien von Valleix, sowie die von Lussanna über Brachialneuralgien, letztere nach dem sehr ausfuhrlichen Auszuge in den Schmidt'schen Jahrbüchern (Bd. 108. p. 168), darauf hin durchgeseluen habe. Die Monographie von Bergson war mir leider nicht zugänglich.

Dass der Nervus cutaneus brachii internus in Fällen von Brachialneuralgien ziemlich häufig betheiligt sei, erwähnt EuJenburg (1. c. p. 135); dass er jedoch ganz isolirt Sitz einer Neuralgic sein könne, davon schreibt er ebensowenig, wie die übrigen Autoren. 